\title{
Robust scheduling algorithm for Guaranteed Bit Rate services
}

\author{
Huda Adibah Mohd Ramli* \\ Faculty of Engineering, \\ Department of Electrical and Computer Engineering, \\ International Islamic University Malaysia (IIUM), \\ P.O. Box 10, 50728 Kuala Lumpur, Malaysia \\ E-mail: hadibahmr@iium.edu.my \\ ${ }^{*}$ Corresponding author
}

\section{Kumbesan Sandrasegaran}

Faculty of Engineering and Information Technology, University of Technology, Sydney, 15 Broadway,

Ultimo, NSW 2007, Australia

E-mail: Kumbesan.Sandrasegaran@uts.edu.au

\begin{abstract}
This paper proposes a novel packet scheduling algorithm to overcome detrimental effects of channel impairments on the quality of service of delay-sensitive Guaranteed Bit Rate (GBR) services. The proposed algorithm prioritises the packets that require retransmission of Hybrid Automatic Repeat Request (HARQ) users compared to the packets of new users. The packets of new users are scheduled according to the Channel Quality Information (CQI), average throughput and packet delay information. Computer simulations have demonstrated that the proposed algorithm has $22.7 \%$ system capacity improvement over a well-known algorithm. It also tolerates for up to $200 \%$ delay of CQI and reduces the uplink signalling overhead by $150 \%$ compared to the well-known algorithm without compromising the quality of service requirements of the GBR services.
\end{abstract}

Keywords: mobile communication; LTE; long-term evolution; OFDMA; orthogonal frequency division multiple access; CQI; channel quality information; QoS; HARQ; hybrid automatic repeat request; packet scheduling; multimedia; channel impairments; GBR; guaranteed bit rate.

Reference to this paper should be made as follows: Ramli, H.A.M. and Sandrasegaran, K. (2013) 'Robust scheduling algorithm for Guaranteed Bit Rate services', Int. J. Mobile Communications, Vol. 11, No. 1, pp.71-88.

Biographical notes: Huda Adibah Mohd Ramli received her $\mathrm{PhD}$ in Engineering from University of Technology, Sydney (Australia) (2012), MSc in Software Engineering from the University of Technology Malaysia (Malaysia) (2006) and BEng in Electrical and Computer Engineering from International Islamic University Malaysia (Malaysia) (2003). Her current research interest generally focuses on radio resource management in the mobile communication systems specifically on packet scheduling and component carrier selection. 
Kumbesan Sandrasegaran holds a $\mathrm{PhD}$ in Electrical Engineering from McGill University (Canada) (1994), a Masters of Science Degree in Telecommunication Engineering from Essex University (UK) (1988) and a Bachelor of Science (Honours) Degree in Electrical Engineering (First Class) (UZ) (1985). He was a recipient of the Canadian Commonwealth Fellowship (1990-1994) and British Council Scholarship (1987-1988). He is a Professional Engineer (Pr.Eng) and has more than 20 years experience working either as a practitioner, researcher, consultant and educator in telecommunication networks. During this time, he has focused on the planning, modelling, simulation, optimisation, security and management of telecommunication networks.

\section{Introduction}

Major advancement of mobile communication system is expected to result in an increasing demand for high-speed multimedia services among the mobile users. The multimedia services, which consist of Guaranteed Bit Rate (GBR) and non-Guaranteed Bit Rate (non-GBR) services, have competing QoS requirements. For instance, most of the GBR applications are more sensitive to packet delay, whereas most of the non-GBR applications are more sensitive to loss packets. In such mobile communication systems where providing QoS that is comparable to the fixed line services are essential, intelligent design of packet scheduling algorithms becomes crucial. However, this remains as a major challenge since packet scheduling in mobile communication systems is significantly different to that in the fixed line. This is due to the radio propagation environment which subjects to error, imperfect Channel Quality Information (CQI) reports, adequate CQI Reporting Rate (CRR) and expensive radio resources.

Long Term Evolution (LTE) is an emerging mobile communication system that delivers multimedia services using packet switching technology. It uses Orthogonal Frequency Division Multiple Access (OFDMA) for downlink transmission (3GPP, 2009). OFDMA is a multi-carrier access technology that divides the available wide bandwidth into multiple equally spaced and mutually orthogonal sub-carriers (Daoud and Alani, 2009). The minimum downlink LTE transmission unit that can be allocated to a user is referred to as a Resource Block (RB). An RB is made up of 12 sub-carriers of $1 \mathrm{~ms}$ duration (Holma and Toskala, 2009). A number of RBs are available for usage among the downlink LTE users at $1 \mathrm{~ms}$ scheduling interval, but each RB can only be assigned to a single user in each scheduling interval.

Conceptually, there have been numerous packet scheduling algorithms developed to provide satisfactory QoS for the multimedia services in the downlink LTE systems. However, study of the literature indicates a number of research gaps which are described next. Majority of the packet scheduling algorithms developed for the downlink LTE mainly focus on providing satisfactory QoS for the delay-tolerant non-GBR services (Assaad and Mourad, 2008; Fattah and Alnuweiri, 2009; Kwan et al., 2008; Tran and Eltawil, 2012; Yuanye et al., 2010). There remains a gap in which these packet scheduling algorithms may be incapable for simultaneous support of multimedia services having competing QoS requirements. Another research gap is that, majority of the developed packet scheduling algorithms assume absence of mobile communication channel impairments (Gidlund and Laneri, 2008; Shih-Jung and Liou, 2011; Siomina and 
Wanstedt, 2008; Sueng Jae et al., 2011; Yan and Guangxin, 2008). This is not a practical assumption as the downlink LTE channels may be impaired due to interference, multi-path fading, shadowing, imperfect CQI reports, CRR, etc. (Capozzi et al., 2012).

Given the stated challenges and the research gaps, as well as a good portion of the radio resources has to be provided by the LTE system in supporting GBR services, this paper presents a new packet scheduling algorithm known as Robust and Quality of service-Driven Scheduling (RQ-DS). This algorithm aims to provide support for the delay-sensitive GBR services over the error-prone downlink LTE channels. Impact of the outdated CQI reports and CRR on RQ-DS performance at different user speeds is extensively studied. The RQ-DS algorithm attempts to address the following research questions: In the presence of mobile communication channel impairments, how is it possible to overcome its detrimental effects on the QoS of GBR services? Do new packet scheduling algorithms need to be developed to overcome detrimental effects caused by these impairments? If so, how much performance improvements do these new algorithms offer over conventional packet scheduling algorithms?

The remaining sections of this paper are organised as follows. The reviews on the related studies are provided in Section 2 followed by detailed descriptions of the RQ-DS algorithm in Section 3. The environment to evaluate the performance of the RQ-DS is described in Section 4. Section 5 contains performance comparisons of the RQ-DS against a well-known packet scheduling algorithm. Conclusions are summarised in Section 6.

\section{Related studies}

Given that channel impairments of mobile communication may cause detrimental effects on the QoS of multimedia services, a large number of packet scheduling algorithms have been developed to address this situation. For example, Wang and Yang (2003) have developed a packet scheduling algorithm that treats packets of users with first transmission differently compared to packets of users that require retransmission. The developed algorithm uses normalised data rate which results in Transport Blocks (TBs) of Hybrid Automatic Repeat Request (HARQ) users being prioritised compared to TBs of new users. Note that TB refers to a group of packets that are (re)transmitted to a user in a scheduling interval. In addition to that, users with TBs of first transmission are referred to as new users, whereas users with retransmitted TBs are referred to as HARQ users, throughout the paper. A packet scheduling algorithm that selects a user on the basis of retransmission delay was developed by Yuanye et al. (2010), whereas Liu et al. (2010) developed an optimisation technique that uses different formulations for TB (re)transmissions to the new and HARQ users.

Pokhariyal et al. (2007) developed an algorithm that decomposes packet scheduling into Time Domain (TD) and Frequency Domain (FD) schedulers. This algorithm is referred to as HARQ aware Frequency Domain Packet Scheduling (HARQ FDPS) in this paper. The TD scheduler uses an algorithm such as the well-known Proportional Fair (PF) (Jalali et al., 2000) algorithm to limit the number of new and HARQ users for the FD scheduler. Thereafter, the FD scheduler reserves an amount of radio resources for the HARQ users, and consequently assigning remaining radio resources to the new users on the basis of a packet scheduling algorithm (i.e., PF algorithm). Similarly, the required 
amount of radio resources is assigned to the HARQ users according to a packet scheduling algorithm.

The algorithms previously discussed were developed under the assumption that users do not have strict requirements concerning packet delay. Therefore, these algorithms are mostly suited for providing satisfactory QoS to the delay-tolerant non-GBR services. A good portion of the radio resources has to be provided to support GBR services that have stringent delay requirements. Therefore, an algorithm with two (2) new features was developed by Ramli et al. (2011). Firstly it extends the well-known Maximum-Largest Weighted Delay First (M-LWDF) (Andrews et al., 2001) algorithm when scheduling packets of new users and secondly, it schedules packets of HARQ users according to the $\mathrm{PF}$ algorithm in combination with a scaling factor that is dependent upon packet delay information. The algorithm gives higher priority for TB retransmissions to the HARQ users as compared to the new users since packets of HARQ users are more likely to reside longer within the buffers at the base station.

Majority of the aforementioned algorithms made common assumptions that either perfect CQI report is available at the base station or this CQI is reported at a high reporting rate which indicates better accuracy of the Modulation and Coding Scheme (MCS) being used. These assumptions are unrealistic within practical mobile communication systems. Though there exist a number of algorithms that were developed under much more realistic assumptions (i.e., Nonchev et al., 2011; Piro et al., 2011; Turyagyenda et al., 2012), the impact of imperfect CQI reports and the CRR towards the performance of the developed algorithms is not extensively investigated.

The impact of the outdated CQI reports on a mobile communication performance was investigated by Martin-Sacristan et al. (2007). The authors showed that the outdated CQI reports lead to degradation in packet scheduling performance. This is due to the rapid channel variation which results in difference between the CQI experienced by user when TB is received and the CQI used to determine MCS for the TB before it is transmitted. Morales-Jimenez et al. (2009) studied the impact of channel estimation errors, outdated and erroneous CQI reports on performance of a multi-carrier mobile communication system. Based on the study, the authors suggested that the system performance is more sensitive to the outdated CQI reports compared to other CQI imperfectness. The impact of the throughput performance due to different sources of CQI errors (i.e., channel estimation error, interference variation and user speed) within Adaptive Modulation and Coding/Hybrid Automatic Repeat Request (AMC/HARQ) systems was studied by $\mathrm{Yu}$ et al. (2010). The authors showed that the CQI errors, particularly those generated based on user's speed, can significantly degrade system performance in a pure AMC system, and those generated by channel estimation are the hardest to recover.

CQI information available at the packet scheduler is more up-to-date when the CQI is reported to the base station at a higher CRR. However, a high CRR comes at the expense of an increase in uplink signalling overhead and thus leads to congestion on feedback channels that are limited in capacity. Therefore, with the aim to limit the CRR from the users, Kim and Lee (2006) developed a CQI reporting method where the CQIs are reported to the base station only by users with instantaneous CQIs above a predefined threshold. The results showed that the CQI reporting method significantly improved the system capacity when erroneous CQI reports are present. Soo-Yong and Dong-Ho (2006) minimised the CRR by proposing a CQI reporting method that allows users to report their CQIs to the base station under two scenarios. Scenario 1 is when the user detects its current CQI is different from the last reported CQI and Scenario 2 is whenever the CQI 
timer located at base station has expired. Note that the CQI timer is updated each time $\mathrm{CQI}$ is reported and once the timer has expired, the base station will request the user to report its CQI. It was shown in the simulation results that the developed method is more efficient in conserving user's battery compared to the method that requires the user to periodically report its CQI.

This paper focuses more on examining impact of CRR towards packet scheduling performance. There is a trade-off between packet scheduling performance and the uplink signalling overhead as a result of the CRR. However, according to Martin-Sacristan et al. (2007), it is possible to optimise the CRR to limit the uplink signalling overhead without compromising the QoS and overall packet scheduling performance. A study which is almost similar to this paper was discussed by Basukala et al. (2010) who studied the performance impact due to different rates in CQI reporting when scheduling GBR users in the downlink LTE. The authors showed that if the number of discarded packets for delay violations (Packet Loss Ratio - PLR) is kept below $10^{-2}$ threshold, then the CQI has to be reported at $10 \mathrm{~ms}$ for a slow-moving user and at $3 \mathrm{~ms}$ for a higher-speed user.

\section{Robust and Quality of Service-Driven Scheduling (RQ-DS)}

This paper proposes RQ-DS algorithm to overcome detrimental effects caused by downlink LTE channel impairments on the QoS of delay-sensitive GBR services. The RQ-DS algorithm is an extension of the well-known M-LWDF algorithm proposed by Andrews et al. (2001). The M-LWDF is chosen as it is one of the well-known algorithms that can provide satisfactory QoS for the GBR services in the single-carrier mobile communication systems. To enable the M-LWDF algorithm to perform packet scheduling in the downlink LTE, this algorithm is adapted such that in each scheduling interval, the adapted M-LWDF algorithm assigns $\mathrm{RB} j$ to user with TB transmission to maximise $\mu_{i, j}(t)$ in the following equation:

$$
\begin{gathered}
\mu_{i, j}(t)=a_{i} \times W_{i} \times \frac{r_{i, j}(t)}{R_{i}(t)} \\
a_{i}=-\frac{\left(\log \delta_{i}\right)}{T_{i}} \\
R_{i}(t)=\left(1-\frac{1}{t_{c}}\right) R_{i}(t-1)+\frac{1}{t_{c}} r \operatorname{tot}_{i}(t) \\
r \operatorname{tot}_{i}(t)=\sum_{j=1}^{\mathrm{RB}_{\max }} I_{i, j}(t) r_{i, j}(t) \\
I_{i, j}(t)=\left\{\begin{array}{l}
1 \quad \text { if packets of user } i \text { are scheduled on RB } j \text { at scheduling interval } t \\
0 \quad \text { if packets of user } i \text { are not scheduled on RB } j \text { at scheduling interval } t
\end{array}\right.
\end{gathered}
$$

where $\mu_{i, j}(t)$ is the priority of user $i$ on $\mathrm{RB} j$ at scheduling interval $t, a_{i}$ is the QoS requirement of user $i, W_{i}(t)$ is the delay of the head-of-line (HOL) packet of user $i$ at scheduling interval $t, r_{i, j}(t)$ is the instantaneous data rate of user $i$ on $\operatorname{RB} j$ at scheduling interval $t, R_{i}(t)$ is the average throughput of user $i$ at scheduling interval $t, \delta_{i}$ is the 
service-dependent PLR threshold of user $i, T_{i}$ is the service-dependent buffer delay threshold of user $i, t_{c}$ is a time constant, $r \operatorname{tot}_{i}(t)$ is the total data rate being used to transmit packets to user $i$ at scheduling interval $t, I_{i, j}(t)$ is the indicator function of the event that packets of user $i$ are selected for transmission on $\mathrm{RB} j$ at scheduling interval $t$ and $\mathrm{RB}_{\max }$ is the maximum available number of RBs. (Note that the HOL packet of user is the packet that has resided the longest in its buffer at base station, whereas the buffer delay threshold is defined as the maximum allowable waiting time of a packet in the buffer at base station (Hua et al., 2011)).

The packets that require retransmission of a HARQ user need to be prioritised as these packets are more likely to reside longer in the buffer at the base station. Furthermore, retransmitted TBs are more likely to be correctly decoded at user's end compared to TBs of the first transmission. This is due to the combining gain of multiple retransmissions. Therefore, compared to the M-LWDF algorithm that gives equal opportunity for TB (re)transmissions between the HARQ and new users, the proposed RQ-DS algorithm gives higher priority for TB retransmissions to the HARQ users and schedules packets of the new users according to the adapted M-LWDF algorithm.

The RQ-DS algorithm is developed under a constraint in which retransmitted TB has to use the same MCS and the same number of RBs as in the first transmission (Kela et al., 2008). Figure 1 shows the flow chart of the RQ-DS algorithm where it performs a maximum of four steps in each scheduling interval. Detailed description of each step is discussed below.

\subsection{Step 1}

At scheduling interval $t$, Step 1 of the RQ-DS is taken to determine the number of RBs required by each HARQ user (i.e., $n \mathrm{HARQ}_{i}(t)$ where symbol $i$ is used to refer to user $i$ at scheduling interval $t$ ), to update a list of retransmitting HARQ users and to calculate the total number of RBs required by all HARQ users (i.e., $\mathrm{RB}_{\mathrm{HARQ}}$ ). This step ensures sufficient number of RBs is allocated to each HARQ user for TB retransmission.

\subsection{Step 2}

Step 2 is executed if $R B_{\text {HARQ }}>R^{\max }$ after Step 1 has been completed. The situation where $\mathrm{RB}_{\mathrm{HARQ}}>\mathrm{RB}_{\max }$ implies that insufficient number of $\mathrm{RBs}$ is available to schedule all of the HARQ users after completion of Step 1. Therefore, one or more HARQ users need to be removed from the list of retransmitting HARQ users. This step gives a random priority to each HARQ user and consequently sorts the users in ascending order of priority. Then, it iterates over HARQ users in their prioritised order. Step 2 removes a HARQ user in turn from this list and reduces $\mathrm{RB}_{\mathrm{HARQ}}$ according to the number of RBs required by the HARQ user. The iteration over HARQ user continues until $\mathrm{RB}_{\text {HARQ }} \leq \mathrm{RB}_{\max }$. HARQ user in the list of retransmitting HARQ users is referred to as retransmitting HARQ user hereafter.

\subsection{Step 3}

Step 3 is executed if remaining $R B s\left(R_{\text {rem }}\right)$ are still available after Step 1 or Step 2 has been completed. This step assigns $\mathrm{RB}_{\mathrm{rem}} \mathrm{RBs}$ for transmission of TBs to the new users. It is not necessary to assign good RBs to the HARQ user as retransmitted TB is more 
likely to be correctly decoded at the user's end. Therefore, scheduling of the retransmitting HARQ users take place after Step 3 has been completed. Step 3 is divided into Part 1 and Part 2. Part 1 generates a channel matrix consisting of $N_{\text {new }}$ users and $\mathrm{RB}_{\max } \mathrm{RBs}$. Each element in the channel matrix is computed according to the adapted M-LWDF algorithm (see equation (1)).

Figure 1 Flow chart of the RQ-DS algorithm in each scheduling interval (see online version for colours)

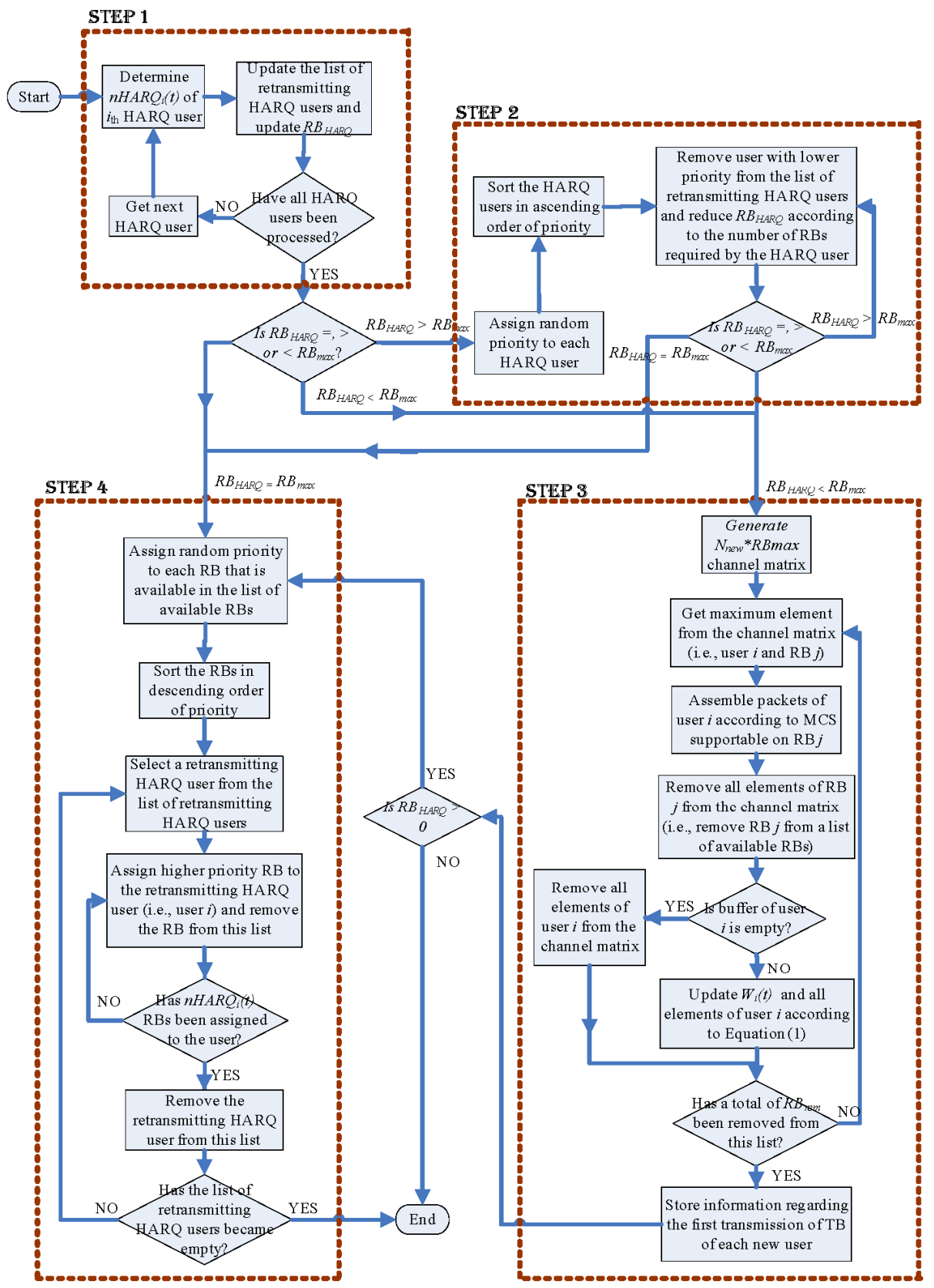


Part 2 selects an element with the maximum value from the channel matrix (i.e., $E_{i, j}(t)$ is the element with the maximum value). This indicates that the throughput of user $i$ can be maximised if its packets are scheduled on $\mathrm{RB} j$. Subsequently, this part assembles the packets of the user according to the MCS supportable on $\mathrm{RB} j$ and removes all elements of $\mathrm{RB} j$ from the channel matrix (i.e., remove $\mathrm{RB} j$ from a list of available RBs). All elements of user $i$ are deleted from the channel matrix if its buffer has become empty after assembling packets for transmission. Otherwise, this part updates new $W_{i}(t)$ and all elements of user $i$ according to equation (1). Part 2 is repeated until a total of $R_{\text {rem }} R B s$ have been removed from this list. Note that user may be assigned one or more RBs in each scheduling interval, and a group of packets that are to be transmitted to the user on these assigned RBs is called TB.

Step 3 stores information regarding the first transmission of TB for each new user (i.e., total number of RBs that is assigned to the new user and MCS supportable on each assigned $\mathrm{RB}$ ). This information will be used if TB is erroneously received by the user and requires retransmission in the subsequent scheduling intervals.

\subsection{Step 4}

Step 4 assigns $\mathrm{RB}_{\mathrm{HARQ}} \mathrm{RBs}$ to the retransmitting HARQ users. This step is executed if $R_{\text {HARQ }}>0$ in Step 1. This implies that one or more HARQ users require TB retransmissions. Random assignment of RBs to the retransmitting HARQ users may lead to improvement in throughput performance as discussed by Soundarajan et al. (2009). Therefore, Step 4 randomly assigns required number of RBs to retransmitting HARQ user and removes the assigned RBs from the list of available RBs. This step is repeated until the list becomes empty (i.e., all retransmitting HARQ users have been assigned their required number of RBs). The TB of each HARQ user is then retransmitted using the same MCS as in the first transmission.

\section{Simulation environments}

In this paper, the downlink LTE contains a single hexagonal cell with a base station located at the centre of the cell. Users are uniformly located within the cell. The base station uses a total of $43.01 \mathrm{dBm}$ transmit power. Only the Frequency Division Duplex (FDD) mode with a $5 \mathrm{MHz}$ transmission bandwidth and $2 \mathrm{GHz}$ carrier frequency is considered. A total of $25 \mathrm{RBs}$ are available to be shared among the users.

A total of 16 CQI values based on 3GPP recommendation (3GPP, 2011) is used. A number of assumptions are made so as to reduce the complexity of the computer simulation. These assumptions are consistent with other studies in the literature (Basukala et al., 2010; Morales-Jimenez et al., 2009). It is assumed that transmitted TBs are subject to errors, the CQI reports that are available at the base station may be imperfect, the CQI is not reported to the base station in each scheduling interval, the base station continuously uses the latest correctly received CQI whenever an up-to-date CQI report is not available and the base station directly uses an outdated CQI reported by a user. Type II HARQ with Chase Combining (CC) (Chase, 1985) that requires the packet scheduler to retransmit an identical TB similar to the first transmission is considered. The HARQ feedback is modelled error-free with a $4 \mathrm{~ms}$ delay. All packets of an erroneous TB are discarded after they have been retransmitted three times. 
It is assumed that all GBR users run video streaming application with average data rates of $256 \mathrm{kbps}$ (Ramli et al., 2011). The buffer delay threshold of the GBR service is capped at $80 \mathrm{~ms}$. A practical GBR PLR threshold of $10^{-3}$ based on 3GPP recommendation is employed (3GPP, 2009). Additionally, it is assumed that minimum user throughput is to be maintained above $235 \mathrm{kbps}$ so as to allow the user to run $2 \mathrm{~min}$ video streaming session without its buffer running dry (if the size of de-jitter buffer is assumed to be $10 \mathrm{~s}$ when the user starts its session).

The performances of RQ-DS are evaluated in terms of PLR and mean user throughput metrics. These metrics are defined as follows:

$$
\begin{aligned}
& \operatorname{PLR}=\frac{\sum_{i=1}^{N} \sum_{t=1}^{T} p \operatorname{discard}_{i}(t)}{\sum_{i=1}^{N} \sum_{t=1}^{T} p \operatorname{size}_{i}(t)} \\
& \text { Mean user throughput }=\frac{1}{N} \frac{1}{T} \sum_{i=1}^{N} \sum_{t=1}^{T} p \operatorname{rrx}_{i}(t)
\end{aligned}
$$

where $p \operatorname{discard}_{i}(t)$ is the total size of discarded packets (in bits) of user $i$ at time $t, p \operatorname{size}_{i}(t)$ is the total size of all packets (in bits) arrive into the base station buffer of user $i$ at time $t$, $\operatorname{prx}_{i}(t)$ is the total size of correctly received packets (in bits) of user $i$ at time $t, N$ is the total number of users and $T$ is the total simulation time.

\section{Results and discussions}

The conventional HARQ FDPS algorithm (Pokhariyal et al., 2007) discussed in Section 2 is used to quantify performance improvement of the RQ-DS algorithm. The HARQ FDPS is one of the well-known packet scheduling algorithms developed to provide support for TB (re)transmission within multi-carrier mobile communication systems (Lima et al., 2010; Vukadinovic and Karlsson, 2010). TD and FD schedulers of the HARQ FDPS use the PF and multi-carrier PF algorithms, respectively, in this performance comparison. The following sub-sections discuss results obtained via system level simulations for the RQ-DS and HARQ FDPS algorithms.

\subsection{Performance comparison with increasing system capacity}

Figures 2 and 3 show the PLR and mean user throughput of both algorithms with increasing system capacity, respectively. In this performance comparison, the speed of each user is fixed at $3 \mathrm{~km} / \mathrm{h}$, the CQI delay is set to $0 \mathrm{~ms}$ and the interval for CQI reporting (CQI Reporting Interval - CRI) is set to $1 \mathrm{~ms}$ interval.

To satisfy the QoS requirements of the GBR services, PLR should be kept below $10^{-3}$ threshold. It is shown in Figure 2 that the maximum system capacities where the QoS requirement of the GBR services is satisfied in the HARQ FDPS and RQ-DS algorithms are 22 and 27 users, respectively. This is equivalent to $22.7 \%$ improvement in the system capacity achieved in the RQ-DS algorithm over the HARQ FDPS algorithm (as shown in Table 1). From Table 2, it is observed that at the maximum system capacity, the RQ-DS algorithm is capable of improving the mean user throughput by $7.7 \%$ over the HARQ FDPS algorithm. In addition to that, the minimum throughput requirement of $235 \mathrm{kbps}$ is maintained by the RQ-DS algorithm for higher system capacity. 
The system capacity improvement achieved in the RQ-DS algorithm is significant since all users stream their video packets with an average data rate of $256 \mathrm{kbps}$. Further benefits of the RQ-DS algorithm in minimising performance degradations due to the impact of outdated CQI reports and the CRR are described next.

Figure 2 PLR vs. system capacity (see online version for colours)

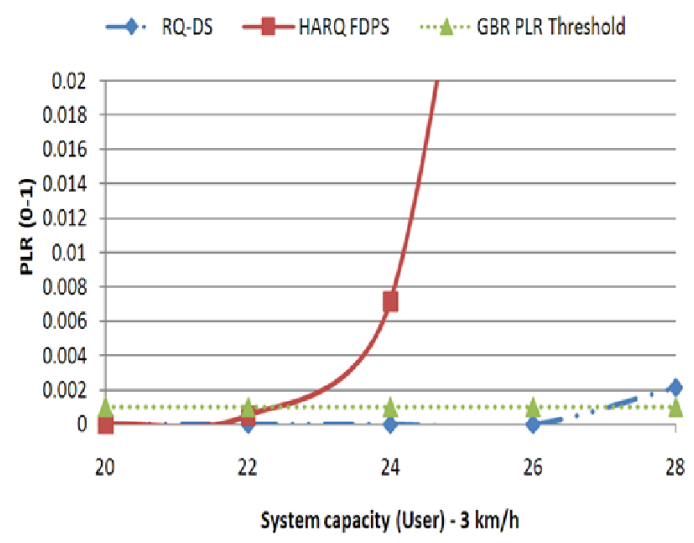

Figure 3 Mean user throughput vs. system capacity (see online version for colours)

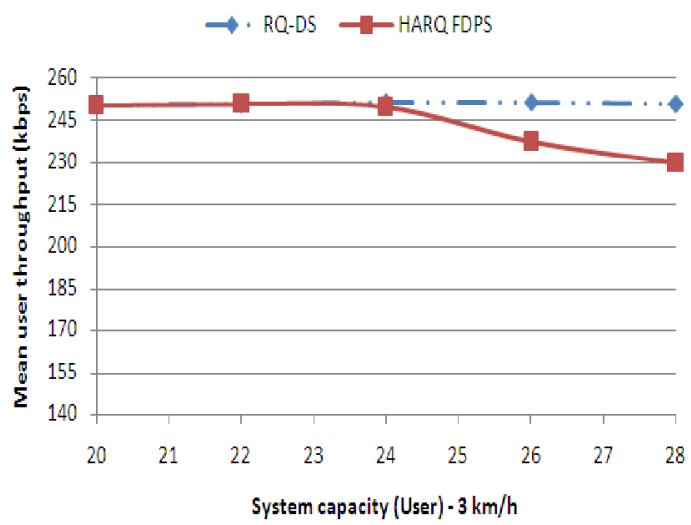

Table 1 Maximum system capacity to satisfy the QoS requirement of the GBR services

\begin{tabular}{lcc}
\hline & & $\begin{array}{c}\text { Percentage of improvement in } \\
\text { RQ-DS over HARQ FDPS }(\%)\end{array}$ \\
\hline HARQ FDPS & 22 & 22.7 \\
RQ-DS & 27 & \\
\hline
\end{tabular}

Table 2 Mean user throughput at 27 users (maximum system capacity achieved in RQ-DS)

\begin{tabular}{lcc}
\hline & $\begin{array}{c}\text { Mean user throughput } \\
(\mathrm{kbps})\end{array}$ & $\begin{array}{c}\text { Percentage of improvement in } \\
\text { RQ-DS over HARQ FDPS }(\%)\end{array}$ \\
\hline HARQ FDPS & 233 & 7.7 \\
RQ-DS & 251 & \\
\hline
\end{tabular}




\subsection{Impact of outdated CQI reports on GBR performance}

The impact of performance due to outdated CQI reports on the PLR and mean user throughput at $3 \mathrm{~km} / \mathrm{h}$ and $30 \mathrm{~km} / \mathrm{h}$ user speeds are illustrated in Figures 4 and 5 , respectively. In this performance comparison, the system capacity is fixed at 20 users (i.e., the system capacity in which the QoS requirement of the GBR services is fulfilled in both algorithms - see Figure 2) and CRI is set to $1 \mathrm{~ms}$ interval.

Figure 4 PLR vs. CQI delay: (a) $3 \mathrm{~km} / \mathrm{h}$ and (b) $30 \mathrm{~km} / \mathrm{h}$ (see online version for colours)

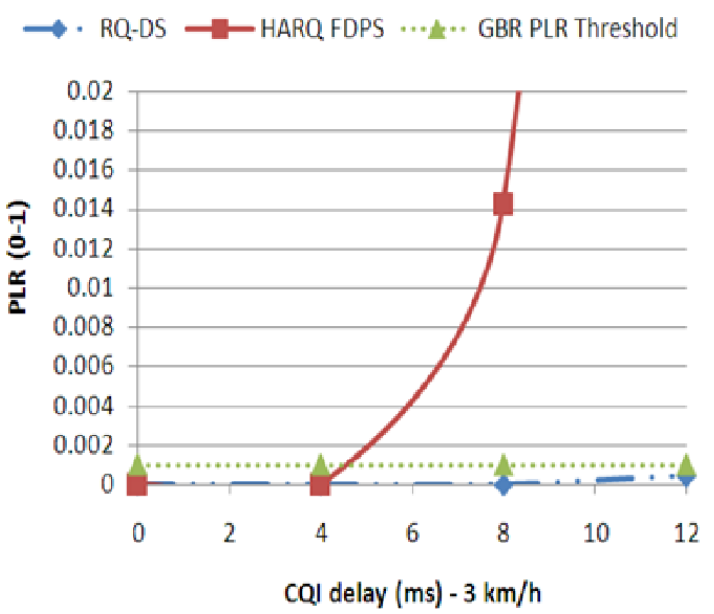

(a)

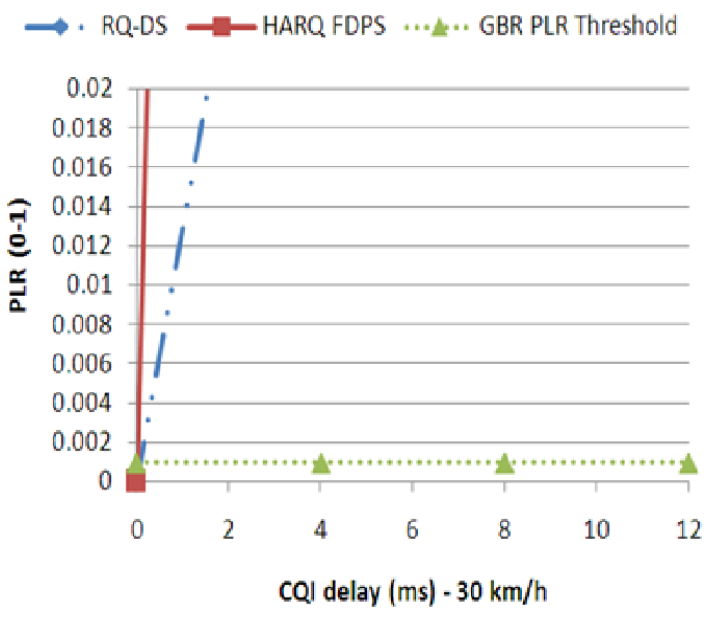

(b)

As expected, more TBs are received in error at the users' end with increasing CQI delay. This is because the CQI experienced by user when TB is received and the CQI that was used to determine the MCS for the TB before it was transmitted is different particularly for user with higher speed due to rapid channel variations. Figures 4(a) and 5(a) illustrate that the outdated CQI reports within the range of 0-12 ms have negligible impact on the 
RQ-DS performances at $3 \mathrm{~km} / \mathrm{h}$ user speed. It can be observed in Figures 4(b) and 5(b) that the PLR and mean user throughput of the RQ-DS and HARQ FDPS algorithms at $30 \mathrm{~km} / \mathrm{h}$ user speed significantly degrade with increasing CQI delay. These degradations are mostly due to the packets being discarded for delay violations and exceeding maximum number of retransmissions.

Figure 5 Mean user throughput vs. CQI delay: (a) $3 \mathrm{~km} / \mathrm{h}$ and (b) $30 \mathrm{~km} / \mathrm{h}$ (see online version for colours)

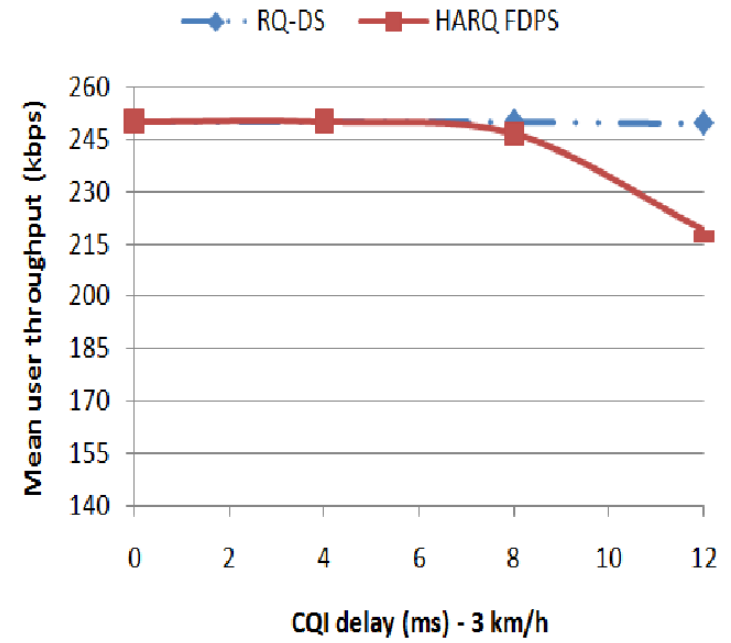

(a)

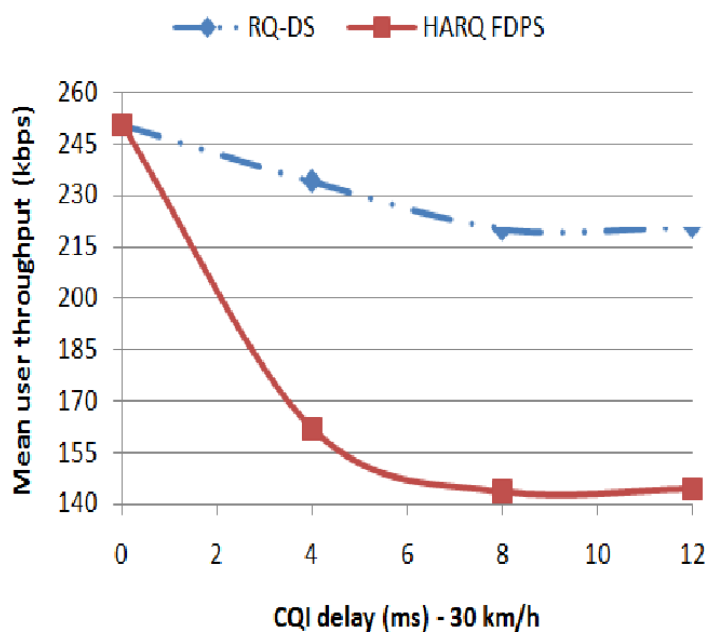

(b)

If the QoS requirement of the GBR services (PLR $<10^{-3}$ ) is to be satisfied at the $3 \mathrm{~km} / \mathrm{h}$ user speed, then the maximum tolerable CQI delay can be as high as $12 \mathrm{~ms}$ in the RQ-DS algorithm and limited to $4 \mathrm{~ms}$ in the HARQ FDPS algorithm (see Table 3). This shows that the RQ-DS performance is more robust to the impact due to the outdated CQI reports compared to the HARQ FDPS. This is evident from Table 3 that shows the RQ-DS 
algorithm can tolerate for up to $200 \%$ CQI delay compared to the HARQ FDPS algorithm.

Table 3 Maximum tolerable CQI delays at $3 \mathrm{~km} / \mathrm{h}$ user speed to maintain the PLR below $10^{-3}$ practical threshold

\begin{tabular}{lcc}
\hline & $\begin{array}{c}\text { Maximum tolerable CQI } \\
\text { delay }(\mathrm{ms})\end{array}$ & $\begin{array}{c}\text { Percentage of improvement in } \\
\text { RQ-DS over HARQ FDPS }(\%)\end{array}$ \\
\hline HARQ FDPS & 4 & 200 \\
RQ-DS & 12 & \\
\hline
\end{tabular}

Furthermore, it is shown in Figure 5(b) that at $30 \mathrm{~km} / \mathrm{h}$ user speed, the minimum throughput of $235 \mathrm{kbps}$ is maintained by RQ-DS algorithm for higher CQI delay (i.e., $4 \mathrm{~ms}$ CQI delay). Table 4 shows that, at $4 \mathrm{~ms}$ CQI delay, the RQ-DS algorithm has $45 \%$ improvement in the mean user throughput compared to the HARQ FDPS algorithm.

Table 4 Mean user throughput at $4 \mathrm{~ms}$ CQI delay at $30 \mathrm{~km} / \mathrm{h}$ user speed

\begin{tabular}{lcc}
\hline & Mean user throughput & $\begin{array}{c}\text { Percentage of improvement in } R Q-D S \\
\text { over HARQ FDPS }(\%)\end{array}$ \\
\hline HARQ FDPS & 162 & 45 \\
RQ-DS & 235 & \\
\hline
\end{tabular}

\subsection{Impact of CRR on GBR performance}

The impact of performance due to the CRR on PLR and mean user throughput at $3 \mathrm{~km} / \mathrm{h}$ and $30 \mathrm{~km} / \mathrm{h}$ user speeds are shown in Figures 6 and 7, respectively. In this performance comparison, the system capacity is fixed at 20 users and the CQI delay is set to $0 \mathrm{~ms}$. It is expected that more TBs are likely to be received in error with increasing CRI. A high CRI indicates that the CQI is reported at a lower rate. As the CRR is directly related to the accuracy of the MCS, a lower CRR may lead to more inaccurate MCS as up-to-date CQI information is not available at the base station.

Figures 6(a) and 7(a) show that the RQ-DS algorithm has a comparable performance to the HARQ FDPS algorithm for slow-moving users. However, when the user's speed is fixed at $30 \mathrm{~km} / \mathrm{h}$, the RQ-DS performance is significantly superior to the HARQ FDPS algorithm for the given CRIs. Tables 5 and 6 show that when the CQI is reported at $5 \mathrm{~ms}$ intervals and the user's speed is fixed at $30 \mathrm{~km} / \mathrm{h}$, the RQ-DS algorithm has $89 \%$ and $7 \%$ improvements in the PLR and the mean user throughput, respectively, compared to the HARQ FDPS algorithm.

Additionally, it is illustrated in Figure 6(b) that users travelling at $30 \mathrm{~km} / \mathrm{h}$ speed need to report their CQI at $1 \mathrm{~ms}$ intervals in the HARQ FDPS algorithm to maintain a PLR below the threshold, whereas the RQ-DS algorithm limits this CQI reporting to $3 \mathrm{~ms}$ intervals. If the minimum throughput of $235 \mathrm{kbps}$ is required to be maintained at $30 \mathrm{~km} / \mathrm{h}$ of user's speed, then each user needs to report its CQI at $4 \mathrm{~ms}$ and $10 \mathrm{~ms}$ intervals in HARQ FDPS and RQ-DS algorithms, respectively (see Table 7). This is equivalent to $150 \%$ reduction in the uplink signalling overhead achieved in the RQ-DS over the HARQ FDPS algorithm. 
Figure 6 PLR vs. CRI: (a) $3 \mathrm{~km} / \mathrm{h}$ and (b) $30 \mathrm{~km} / \mathrm{h}$ (see online version for colours)

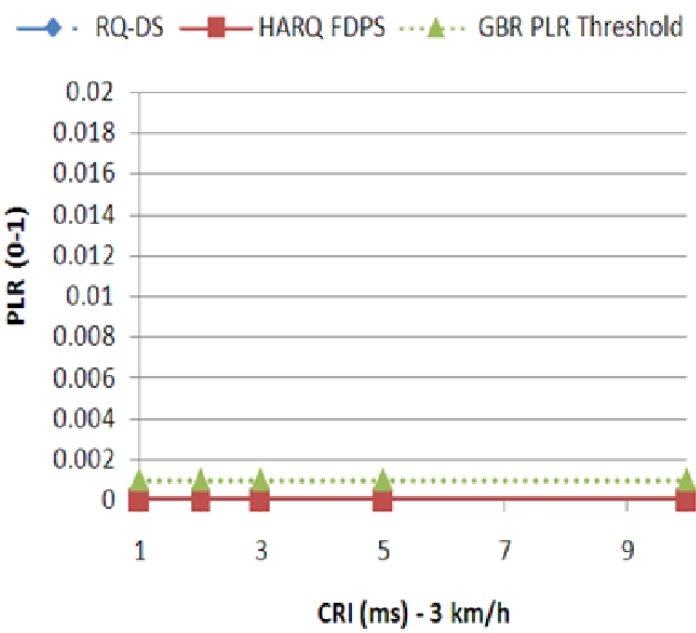

(a)

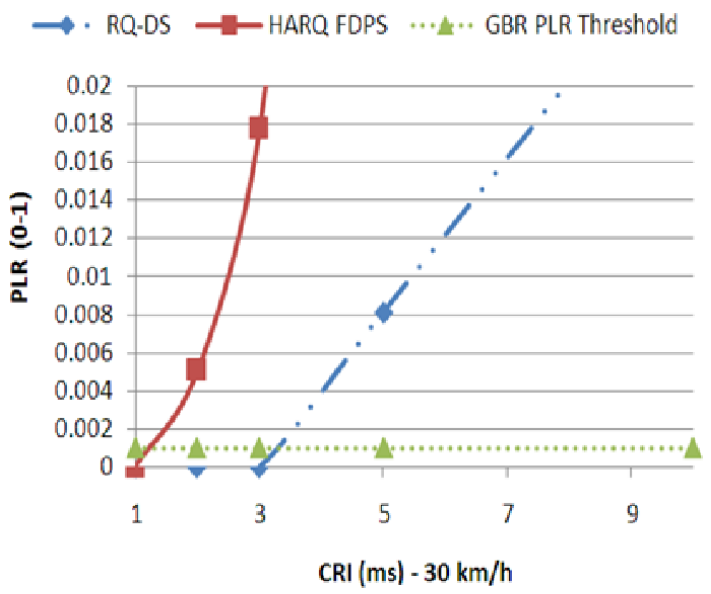

(b)

Table 5 PLR for $5 \mathrm{~ms}$ CRI and at $30 \mathrm{~km} / \mathrm{h}$ user speed

\begin{tabular}{lcc}
\hline & $P L R$ & $\begin{array}{c}\text { Percentage of improvement in } \\
\text { RQ-DS over HARQ FDPS }(\%)\end{array}$ \\
\hline HARQ FDPS & 0.071704 & 89 \\
RQ-DS & 0.008104 & \\
\hline
\end{tabular}

It can be concluded based on the results that the RQ-DS algorithm is more efficient than the HARQ FDPS algorithm in providing satisfactory QoS for the delay-sensitive GBR services in the downlink LTE. As the packets of the HARQ users are more likely to reside longer in the buffers at base station, the RQ-DS algorithm always gives higher priority for TB retransmissions to the HARQ users such that the PLR can be minimised. Compared to the HARQ FDPS algorithm that does not consider packet delay, scheduling 
packets of the new users according to the adapted M-LWDF algorithm allow the RQ-DS to further improve system performance. Note that the M-LWDF algorithm gives higher priority for TB transmission to the new user with packets that reside longer in the buffer at the base station.

Figure 7 Mean user throughput vs. CRI: (a) $3 \mathrm{~km} / \mathrm{h}$ and (b) $30 \mathrm{~km} / \mathrm{h}$ (see online version for colours)

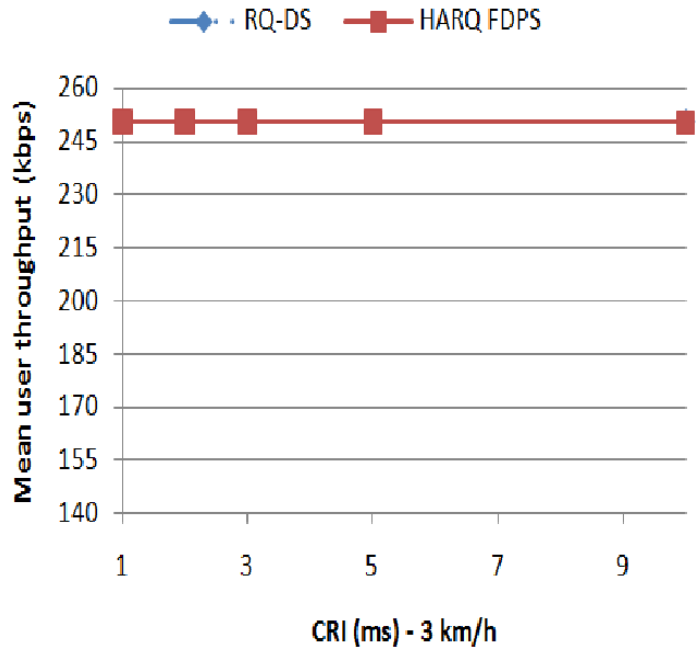

(a)

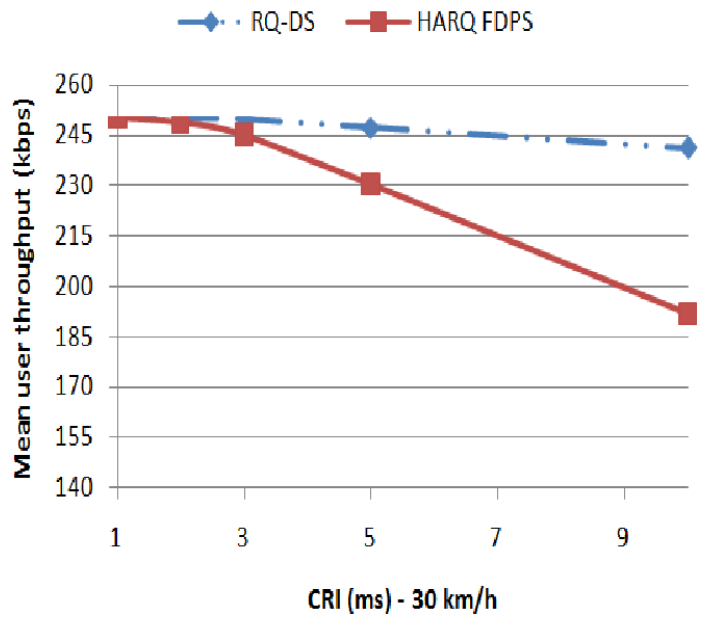

(b)

Table 6 Mean user throughput for $5 \mathrm{~ms} \mathrm{CRI}$ and at $30 \mathrm{~km} / \mathrm{h}$ user speed

\begin{tabular}{lcc}
\hline & $\begin{array}{c}\text { Mean user throughput } \\
(\mathrm{kbps})\end{array}$ & $\begin{array}{c}\text { Percentage of improvement in } \\
\text { RQ-DS over HARQ FDPS }(\%)\end{array}$ \\
\hline HARQ FDPS & 230 & 7 \\
RQ-DS & 247 & \\
\hline
\end{tabular}


Table 7 CRIs at $30 \mathrm{~km} / \mathrm{h}$ user speed to maintain $235 \mathrm{kbps}$ minimum throughput

\begin{tabular}{lcc}
\hline & CRI & $\begin{array}{c}\text { Percentage of improvement in } \\
\text { RQ-DS over HARQ FDPS }(\%)\end{array}$ \\
\hline HARQ FDPS & 4 & 150 \\
RQ-DS & 10 & \\
\hline
\end{tabular}

\section{Conclusions}

This paper proposed an algorithm known as RQ-DS to overcome detrimental effects due to downlink LTE channel impairments on the QoS of delay-sensitive GBR services. The RQ-DS algorithm prioritises the packets that require retransmission of HARQ users compared to the packets of new users. This is due to packets of HARQ users are more likely to reside longer in the buffers at the base station and retransmitted TBs are more likely to be correctly decoded at users' end compared to TBs of the first transmission due to combining gain of multiple retransmissions. The packets of new users are scheduled in the RQ-DS according to the adapted M-LWDF algorithm.

A series of system level simulations was conducted to evaluate RQ-DS performance. With the PLR kept below $10^{-3}$ practical threshold and the average data rate of all users are fixed at $256 \mathrm{kbps}$, the RQ-DS has $22.7 \%$ system capacity improvement over HARQ FDPS algorithm. The RQ-DS and HARQ FDPS algorithms are sensitive against the outdated CQI reports. However, the RQ-DS algorithm is superior to that of HARQ FDPS algorithm in minimising the impact on performance due to the outdated CQI reports. It was shown in the results that the RQ-DS can tolerate for up to $200 \%$ CQI delay compared to the HARQ FDPS without compromising the QoS requirements of the GBR services. Moreover, the RQ-DS algorithm is more efficient in minimising the uplink signalling overhead. Should the minimum throughput of $235 \mathrm{kbps}$ is required to be maintained, the RQ-DS reduces the uplink signalling overhead by $150 \%$ compared to the HARQ FDPS algorithm.

The proposed algorithm can benefit service providers in a number of ways. Firstly, the RQ-DS algorithm allows the service providers to provide support for more users for a given bandwidth and therefore maximising their revenue. This is evident from the simulation results that showed a significant system capacity improvement being achieved under the proposed algorithm. Secondly, the RQ-DS algorithm is efficient in minimising the uplink signalling overhead in the feedback channel. Finally, the RQ-DS algorithm has a low computational complexity and therefore, can easily be implemented in the downlink LTE, as well as other multi-carrier mobile cellular systems without the additional hardware costs.

The RQ-DS algorithm is developed to provide satisfactory QoS for GBR services in the error-prone downlink LTE channels. Future studies will extend the algorithm so as both GBR and non-GBR services can be simultaneously supported. The impact on performance due to other sources of channel impairments, such as channel estimation errors, erroneous CQI reports, absence in CQI reporting, etc. could also be part of future study. Additionally, the performance of the proposed algorithm can be evaluated in multiple cell scenarios where the effect of inter- and intra-cell interference will be accounted. 


\section{References}

3GPP (2009) TS 23.203 (V9.3.0) Policy and Charging Control Architecture (Release 9), 3GPP.

3GPP (2011) TR 36.213 Physical Layer Procedures (Release 10), 3GPP.

Andrews, M., Kumaran, K., Ramanan, K., Stolyar, A., Whiting, P. and Vijayakumar, R. (2001) 'Providing quality of service over a shared wireless link', IEEE Communications Magazine, Vol. 39, No. 2, pp.150-154.

Assaad, M. and Mourad, A. (2008) 'New frequency-time scheduling algorithms for 3GPP/LTE-like OFDMA air interface in the downlink', IEEE Vehicular Technology Conference, Marina Bay, Singapore, pp.1964-1969.

Basukala, R., Ramli, H.A.M., Sandrasegaran, K. and Chen, L. (2010) 'Impact of CQI feedback rate/delay on scheduling video streaming services in LTE downlink', 12th IEEE International Conference on Communication Technology, Nanjing, China, pp.1349-1352.

Capozzi, F., Piro, G., Grieco, L., Boggia, G. and Camarada, P. (2012) 'Downlink packet scheduling in LTE cellular networks: key design issues and a survey', IEEE Communications Surveys \& Tutorials, pp.1-23.

Chase, D. (1985) 'Code combining - a maximum-likelihood decoding approach for combining an arbitrary number of noisy packets', IEEE Transactions on Communications, Vol. 33, No. 5, pp.385-393.

Daoud, O. and Alani, O. (2009) 'Peak to average power ratio reduction technique for MIMO/OFDM systems', Int. J. Mobile Communications, Vol. 7, No. 6, pp.632-645.

Fattah, H. and Alnuweiri, H. (2009) 'A cross-layer design for dynamic resource block allocation in 3G long term evolution system', IEEE 6th International Conference on Mobile Adhoc and Sensor Systems, Macao, China, pp.929-934.

Gidlund, M. and Laneri, J.C. (2008) 'Scheduling algorithms for 3GPP long-term evolution systems: from a quality of service perspective', IEEE 10th International Symposium on Spread Spectrum Techniques and Applications, Bologna, Italy, pp.14-117.

Holma, H. and Toskala, A. (2009) LTE for UMTS: OFDMA and SC-FDMA Based Radio Access, John Wiley \& Sons, Ltd., UK.

Hua, W., Rosa, C. and Pedersen K. (2011) 'Performance analysis of downlink inter-band carrier aggregation in LTE-advanced', IEEE Vehicular Technology Conference, San Fransisco, USA, pp.1-5.

Jalali, A., Padovani, R. and Pankaj, R. (2000) 'Data throughput of CDMA-HDR a high efficiency-high data rate personal communication wireless system', IEEE 51st Vehicular Technology Conference Proceedings, Tokyo, Japan, Vol. 3, pp.1854-1858.

Kela, P., Puttonen, J., Kolehmainen, N., Ristaniemi, T., Henttonen, T. and Moisio, M. (2008) 'Dynamic packet scheduling performance in UTRA long term evolution downlink', 3rd International Symposium on Wireless Pervasive Computing, Greece, pp.308-313.

Kim, D. and Lee, I-H. (2006) 'On capacity of quality-based channel-state reporting in mobile systems with greedy transmission scheduling', IEEE Transactions on Communications, Vol. 54, No. 6, pp.975-979.

Kwan R., Leung, C. and Zhang, J. (2008) 'Multiuser scheduling on the downlink of an LTE cellullar system', Hindawi Publishing Corporation (Research Letters in Communications), pp.1-4.

Lima, F.R.M., Wanstedt, S., Cavalcanti, F.R.P. and Junior, W.C.F. (2010) 'Scheduling for improving system capacity in multiservice 3GPP LTE', Journal of Electrical and Computer Engineering, Vol. 2010, pp.1-16.

Liu, X., Zhu, H. and Wang, J. (2010) 'Adaptive resource allocation with packet retransmissions in OFDMA systems', IEEE International Conference on Communications (ICC), Cape Town, South Africa, pp.1-5. 
Martin-Sacristan, D., Monserrat, J.F., Gozalvez, J. and Cardona, N. (2007) 'Effect of channelquality indicator delay on HSDPA performance', IEEE 65th Vehicular Technology Conference (Spring), Dublin, Ireland, pp.804-808.

Morales-Jimenez, D., Sanchez, J.J., Gomez, G., Aguayo-Torres, M.C. and Entrambasaguas, J.T. (2009) 'Imperfect adaptation in next generation OFDMA cellular systems', Journal of Internet Engineering, Vol. 3, pp.202-209.

Nonchev, S., Valkama, M. and Hamila, R. (2011) 'Advanced packet scheduling for efficient video support with limited channel feedback on MIMO LTE downlink', IEEE GLOBECOM Workshops, Houston, Texas, USA, pp.766-771.

Piro, G., Grieco, L.A., Boggia, G., Fortuna, R. and Camarda, P. (2011) 'Two-level downlink scheduling for real-time multimedia services in LTE networks', IEEE Transactions on Multimedia, Vol. 13, No. 5, pp.1052-1065.

Pokhariyal, A., Pedersen, K.I., Monghal, G., Kovacs, I.Z., Rosa, C., Kolding, T.E. and Mogensen, P.E. (2007) 'HARQ aware frequency domain packet scheduler with different degrees of fairness for the UTRAN long term evolution', IEEE Vehicular Technology Conference, Dublin, Ireland, pp.2761-2765.

Ramli, H.A.M., Sandrasegaran, K., Basukala, R. and Afrin, T.S. (2011) 'HARQ aware scheduling algorithm for the downlink LTE system', International Conference on Modeling, Simulation and Applied Optimization, Kuala Lumpur, Malaysia, pp.1-4.

Shih-Jung, W. and Liou, C. (2011) 'A novel packet scheduling scheme for downlink LTE system', Seventh International Conference Intelligent Information Hiding and Multimedia Signal Processing (IIH-MSP), China, pp.25-28.

Siomina, I. and Wanstedt, S. (2008) 'The impact of QoS support on the end user satisfaction in LTE networks with mixed traffic', IEEE 19th International Symposium on Personal, Indoor and Mobile Radio Communications, Cannes, France, pp.1-5.

Soo-Yong, J. and Dong-Ho, C. (2006) 'Channel adaptive CQI reporting schemes for UMTS high-speed downlink packet access', IEEE 64thVehicular Technology Conference, Montréal, Québec, Canada, pp.1-5.

Soundarajan, S., Agrawal, P. and Li, Y. (2009) 'An efficient HARQ retransmission algorithms in OFDMA based wireless networks', 41st Southeastern Symposium on System Theory, Tullahoma TN, USA, pp.88-93.

Sueng Jae, B., Bum-Gon, C. and Min Young, C. (2011) 'Delay-aware packet scheduling algorithm for multiple traffic classes in 3GPP LTE system', 17th Asia-Pacific Conference on Communications (APCC), Kota Kinabalu, Malaysia, pp.33-37.

Tran, S.V. and Eltawil, A.M. (2012) 'Optimized scheduling algorithm for LTE downlink system', Wireless Communications and Networking Conference (WCNC), Paris, France, pp.1462-1466.

Turyagyenda, C., O'Farrell, T. and Guo, W. (2012) 'Long term evolution downlink packet scheduling using a novel proportional-fair-energy policy', IEEE 75th Vehicular Technology Conference (VTC Spring), Yokohama, Japan, pp.1-6.

Vukadinovic, V. and Karlsson, G. (2010) 'Video streaming performance under proportional fair scheduling', IEEE Journal on Selected Areas in Communications, Vol. 28, No. 3, pp.399-408.

Wang, Y. and Yang, H. (2003) 'Retransmission priority scheduling algorithm for forward link packet data service', International Conference on Communication Technology Proceedings, Beijing, China, Vol. 2, pp.926-930.

Yan, L. and Guangxin, Y. (2008) 'Channel-adapted and buffer-aware packet scheduling in LTE wireless communication system', 4th International Conference on Wireless Communications, Networking and Mobile Computing, Dalian, China, pp.1-4.

Yu, C-H., Hellsten, A. and Tirkkonen, O. (2010) 'Rate adaptation of AMC/HARQ systems with CQI errors', IEEE 71st Vehicular Technology Conference, Taipei, Taiwan, pp.1-5.

Yuanye, W., Pedersen, K.I., Sorensen, T.B. and Mogensen, P.E. (2010) 'Carrier load balancing and packet scheduling for multi-carrier systems', IEEE Transactions on Wireless Communications, Vol. 9, No. 5, pp.1780-1789. 